\title{
Outsourcing cognitive control to the environment: Adult age differences in the use of task cues
}

\author{
DANIEL H. SPIELER, ULRICH MAYR, and SUSAN LAGRONE \\ Georgia Institute of Technology, Atlanta, Georgia
}

\begin{abstract}
When an initial phase of cued task switching is followed by a phase of single-task trials, older adults show difficulties changing to the more efficient single-task mode of processing (Mayr \& Liebscher, 2001). In Experiment 1, we show that these costs follow older adults' continued tendency to inspect task cues even though these provide no new information. In Experiment 2, we included a condition in which task cues were eliminated from the display after the task-switching phase. In this condition, older adults behaved the same as younger adults, suggesting that the presence of the task cue is critical for observing age differences while switching from a "high-control" to a "low-control" mode of processing. We discuss our results in terms of a life-span shift with regard to the reliance on internal versus external sources of information under conditions of high-control demands.
\end{abstract}

Assume you are about to prepare a meal for friends coming over for dinner. Initially, you will spend some time thinking about necessary ingredients and the exact sequence of steps. This phase requires deliberation and active processing of external information (e.g., from cookbooks). Once the plan is laid out, and assuming everything goes as planned, you should be able to execute the remaining routine activities in a relatively ballistic manner, freeing up mental capacity for other activities such as chatting with your arriving friends. Many day-to-day situations require such a back and forth between high-control and low-control phases.

Ideally, we should enter a low-control mode whenever possible and use the costly high-control mode only when necessary. Are we able to rapidly switch from high-control phases requiring intense, deliberate information processing, to phases in which information intake is geared toward the demands of routine activities?

Normal younger adults appear to have little difficulty in shifting control modes. Using a variant of the taskswitching paradigm, Mayr and Liebscher (2001) required younger and older participants to switch between responding to the color or form of a stimulus on the basis of task cues (color and shape) presented above the stimulus. On the first 40 trials, individuals switched randomly from one task to another, prompted by the cue. This situation requires significant top-down control and active use of the cue to select the relevant task set on each trial. However, from Trial 41 onward, one of the two tasks dropped out,

U.M. and D.S. contributed equally to this article; author sequence was determined by coin toss. This work was supported through NIA Grants R01 AG19296-01A1 and R01 AG17024-01A1 and NIH Institutional Training Grant T32 AG00175. Correspondence concerning this article should be addressed to D. H. Spieler, School of Psychology, 654 Cherry Street, Georgia Institute of Technology, Atlanta, GA 30332-0170 (e-mail: spieler@gatech.edu). and only one task was relevant for the remaining 80 trials. Individuals were informed which task would drop out at the start of the block, and the no-longer-relevant task indicator was "marked out" to emphasize that this task was now irrelevant. Obviously, during this so-called fadeout phase, top-down task selection and task cue use are no longer necessary, and individuals should switch into a low-control mode. Indeed, only 10 trials into the fadeout phase, the younger adults' performance was identical to their performance in a single-task control situation in which only one task was relevant throughout the block. In contrast, older adults showed a large, 300-msec "fade-out" cost (fade-out phase RT minus corresponding single-task RT) after one of the two tasks dropped out, and a 100-msec cost remained at the end of the block. In other words, older adults continued to experience a significant influence of the initial task-selection phase long after younger adults had ceased to show any such influence. These results suggest that the selection of control modes is age sensitive, and that a better understanding of the general problem of control mode selection should result from a closer examination of how older and younger adults transition between control modes.

One potential reason for older adults' failure to transition fully to single-task performance levels is suggested by studies of visual perception (see, e.g., Ballard, Hayhoe, Pook, \& Rao, 1997) that indicate that people encode limited information about the environment, preferring to use the environment as an external memory. The degree to which people rely on internal versus external information sources varies with task demands (Ballard et al., 1997) and may also vary with either objective or subjective quality of internal representations. Task-selection situations appear particularly difficult for older adults (e.g., Kray \& Lindenberger, 2000), because they have problems maintaining reliable representations of competing task sets (Braver et al., 2001; Mayr \& Liebscher, 2001). This dif- 
ficulty may increase older adults' reliance on cue processing to update continually degrading task representations. We hypothesize that the reliance on external information to compensate for noisy internal representations overgeneralizes beyond the phases in which it is necessary. During the initial task-switching phase, older adults may establish a strong visual routine (Ballard et al., 1997) of inspecting the task cue, and this routine persists into the fade-out phase, accounting for the age difference in performance.

A straightforward prediction follows from this account: If older adults fail to shift to a low-control mode due to continued reliance on task cues, they should continue to inspect the task cue during the fade-out phase even though it contains no new information. In Experiment 1, we used the basic paradigm introduced by Mayr and Liebscher (2001), but we used eye-movement analyses to examine how older and younger adults used the task cues during the fade-out phase.

\section{EXPERIMENT 1}

\section{Method}

Participants. Twenty-four healthy older adults with no history of neurological disorders $(M$ age $=71.9, S D=4.5)$ and 24 younger adults $(M$ age $=19.3, S D=1.9)$ recruited from the undergraduate student population at the Georgia Institute of Technology participated. Older adults received $\$ 10 / \mathrm{h}$ for participation; younger adults received course credit. Younger adults scored an average 73.1 (5.2) on the Digit-Symbol subtest of the Wechsler Adult Intelligence Scale (WAIS; Wechsler, 1981) in comparison with 47.1 (10) for older adults.

Apparatus. Eye movements were monitored using an ISCAN ETL-400 (ISCAN, Burlington, MA) remote video-based pupil/corneal reflection system, sampling at $120 \mathrm{~Hz}$. A ViewSonic P815 21-in. monitor displayed stimuli. The participants used a chin/forehead rest to control head movements and to maintain a viewing distance of $81 \mathrm{~cm}$.
Materials. An example stimulus is shown in Figure 1. The color and form task cues were separated by $20.6^{\circ}$, and both were $14.9^{\circ}$ from the $2.7^{\circ} \times 1.1^{\circ}$ vertically or horizontally oriented bars that required either color or form (orientation) judgments from the participants.

Procedure. Sessions took 60 to $90 \mathrm{~min}$. Instructions specific to each block of trials (single task, mixed task, and fade-out blocks) were provided at the start of each block of trials. The actual experiment began only when a participant's eye position calibration error was below $1^{\circ}$. The participants then completed 50 practice trials consisting of five miniblocks mirroring those of the experimental session: single-task trials in color and form judgments, switching trials, and fade-out style trials ending in either color or form tasks, followed by six experimental blocks of 60 trials each. There were two single-task blocks, two switching blocks, and two fade-out blocks.

On each trial, (1) a validation point appeared and required a stable fixation within $1^{\circ}$ for $800 \mathrm{msec}$ before the start of the trial, (2) after the validation criteria had been met, the display appeared, eye position recording began, and the display remained until a response, and (3) after a response, validation for the next trial appeared.

Tasks required discriminations based on the color (red vs. blue) or form (vertical vs. horizontal) of the stimuli. The participants made their responses using the "z" and "/" keys on the keyboard. Saccades were defined as eye movements exceeding $90 \% \mathrm{sec}$ and traveling at least $1^{\circ}$ from beginning to end, whereas all other samples were assumed to be fixations. Fixation location was defined as the mean $x$ and $y$ coordinate from the end of one saccade to the start of the next. If velocity criteria identified fixations lasting less than $50 \mathrm{msec}$, these data points were reassigned to the preceding saccade.

\section{Results}

Incorrect responses and correct response times less than $200 \mathrm{msec}$ or greater than $3,000 \mathrm{msec}$ were eliminated from all of the analyses. Analyses focused on performance during the single-task phase of the fade-out block (i.e., Trials 32-60; henceforth, the fade-out phase) in comparison with the corresponding trials from the single-task blocks. Error rates on the critical fade-out block were below 5\% for both groups, so we did not pursue further analyses of 
error rates. ${ }^{1}$ Trials in the fade-out phase were analyzed in terms of six 5-trial segments (except for the first, 4-trial segment). Unless otherwise noted, all effects were significant at the .01 level.

As shown in Figure 2A, during the mixed phase of the fade-out block, both younger and older adults exhibited large switch costs (i.e., the difference between the switch and nonswitch RTs) and mixing costs (i.e., the difference between the nonswitch RT and the single-task RT). Both the switching and mixing costs were qualitatively identical to those in the full mixed-task blocks. In the fadeout phase, older adults had much larger fade-out costs than younger adults (i.e., fade-out RTs in comparison with corresponding single-task RTs) $[F(1,46)=30.68]$; there was a significant linear decrease in fade-out costs across trial segments $[F(1,46)=88.08]$ and this decrease was larger for older than for younger adults $[F(1,46)=$ 12.19]. However, for the final segment, younger adults' performance was indistinguishable from single-task performance $[t(23)=.52, p>.61 ; 19 \mathrm{msec}]$ whereas older adults' performance was still slower than single-task performance $[t(23)=6.30 ; 299 \mathrm{msec}]$.

As shown in Figure 2B, older adults were much more likely to fixate cues than younger adults $[F(1,46)=61.67]$ (analyses were based on arc-sine transformed probabilities; Winer, Brown, \& Michels, 1991). At the end of the block, the younger adults were no more likely to fixate the cue than in single-task performance $[t(23)<1]$, whereas

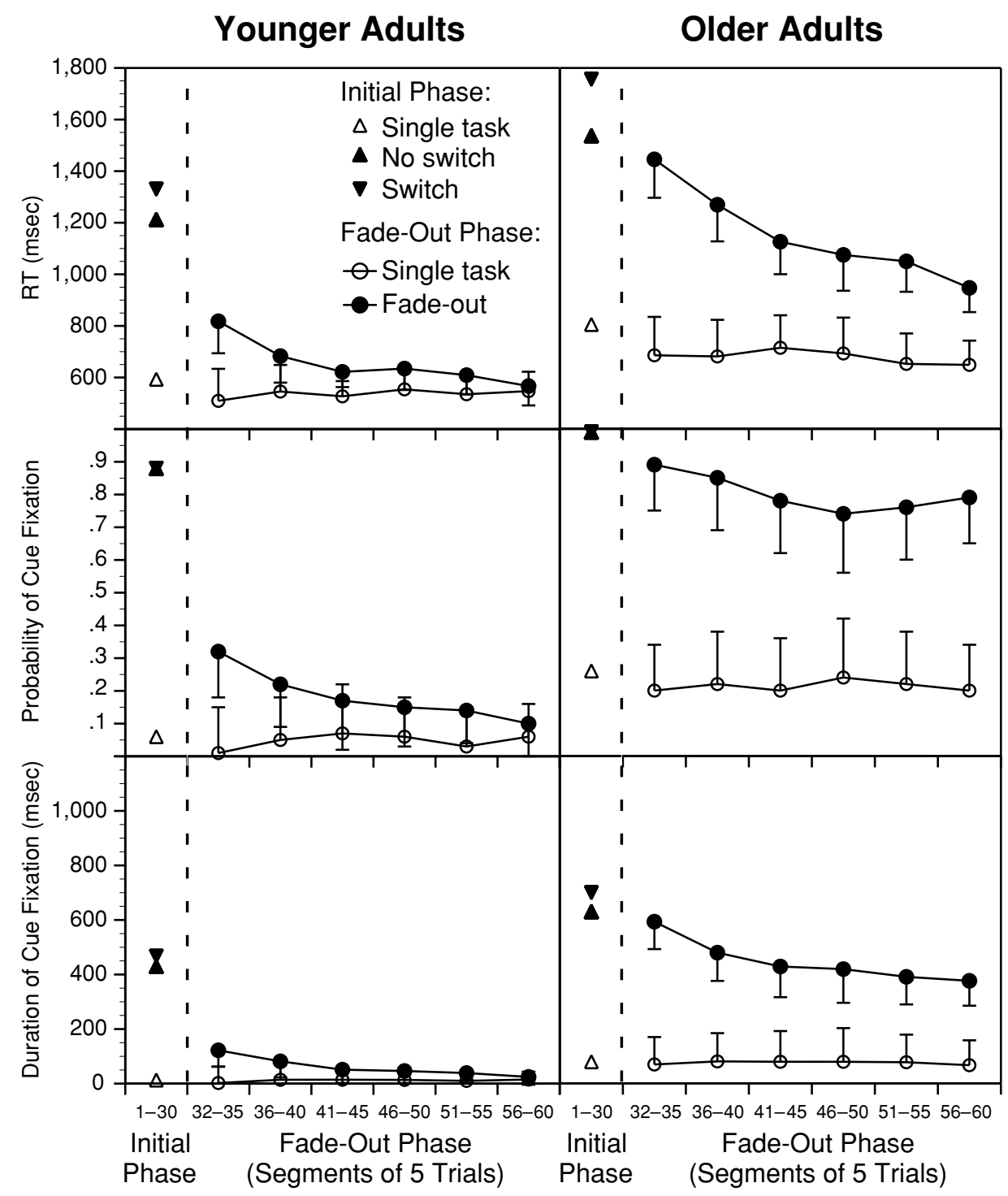

Figure 2. Older and younger adults' mean RTs (top panel), cue-fixation probabilities (middle panel), and average time on cue (bottom panel) for the mixed-task phase and for the five segments of the fade-out phase with corresponding segments of the single-task conditions. Error bars represent the $\mathbf{9 5 \%}$ within-subject confidence interval for the contrast between fade-out and single-task conditions, computed separately for each segment. 
for older adults the critical difference remained highly reliable $[t(23)=8.21]$.

As shown in Figure $2 \mathrm{C}$, the average gaze time on the cue per trial was much larger for older than for younger adults. For the first two fade-out segments, this time was considerably shorter than the corresponding RT fade-out cost (525 msec and $399 \mathrm{msec}$ vs. $760 \mathrm{msec}$ and $588 \mathrm{msec}$ for gaze time and RT, respectively), suggesting that cue processing cannot entirely explain fade-out costs early in the fade-out phase. However, during the remaining four segments, cue-inspection costs were only slightly shorter than RT fade-out costs (349 $\mathrm{msec}, 340 \mathrm{msec}, 313 \mathrm{msec}$, and $310 \mathrm{msec}$ vs. $411 \mathrm{msec}, 382 \mathrm{msec}, 398 \mathrm{msec}$, and 299 msec, respectively).

We computed the correlation between each individual's RT fade-out cost and the mean time per trial each individual spent on the task indicators. Across age groups, this correlation was .81. Within older adults, it was .74, whereas within younger adults, it was only .39 , reflecting the restriction of range arising from the much lower level of cue checks in this group. If the RT cost is directly associated with processing of these task cues, then we should expect a similar relationship to hold within individuals. We regressed the RT for each trial onto time on the task indicators for that trial in the fade-out blocks. We found an average unstandardized slope of 1.4 (young, 1.82; old, 0.99) and an average $R^{2}$ of .34 (young, .31; old, $.37)$, which is a substantial amount of variance that can be explained by the many factors that drive trial-to-trial RTs fluctuations. The slope measure for younger adults should be interpreted with caution, because of the very small number of cue fixations in this group. Combined, these results suggest that cue processing is almost entirely responsible for age differences in performance during the fade-out phase of the experiment.

We hypothesized that older adults use the cue during the fade-out phase in the same way they do during initial task-selection trials - namely, to ensure an adequate task set prior to stimulus processing. Alternatively, older adults may be less confident about their performance and therefore engage a postselection checking stage. This predicts cue inspections late in the trial. In fact, on average, cue inspections occurred after only $22 \%$ of the total time within the trial for older adults and after 33\% of the total time within the trial for younger adults. Thus, particularly for older adults, this pattern is consistent with reliance on cue information prior to processing of the stimulus.

\section{EXPERIMENT 2}

Experiment 1 demonstrates that older adults rely on external information to a far greater extent than younger adults and that RT fade-out cost was strongly related to the time spent on the task cues. Furthermore, cue fixations occurred early in the trial, suggesting that these fixations were reinforcing the current task set rather than being associated with a checking stage just prior to response. These results suggest that older adults maintain a high level of reliance on external information and that this reliance is responsible for the observed age differences in fade-out costs.

An alternative account for these results is that older adults have difficulty in maintaining a consistent task set over time (Braver et al., 2001) and that the reliance on cue information, far from causing these fade-out effects, actually attenuates otherwise larger age differences that would arise in the absence of this cue information.

These two accounts make opposite predictions about the role that task cues play in fade-out cost age differences. In the former account, it is the presence of the cues during the fade-out phase that continues to prompt cuechecking behavior in older adults. Thus, removal of the task cues during the fade-out should improve older adults' performance. In the latter account, the cues are critical in supporting older adults' performance, and cue removal should exacerbate fade-out costs. We tested these contrasting predictions by manipulating the presence of task cues during the fade-out phase of the experiment. We included both the fade-out condition with the cues present, as in Experiment 1, and one in which the cues disappear during the fade-out phase.

\section{Method}

Participants. The participants were 24 older $(M=77.8, S D=$ $6.7)$ and 24 younger $(M=22.0, S D=4.3)$ adults with no history of neurological disorders. Younger adults were recruited from the undergraduate student population at the University of Oregon and participated for course credit. Older adults were recruited from the Eugene, Oregon community and were paid $\$ 10 / \mathrm{h}$ for participation. All of the participants were in good health and had normal or corrected-to-normal vision. On their WAIS Digit-Symbol substitution, the older adults scored an average of 45.6 (11.1) and the younger adults scored 64.9 (9.9).

Materials. Stimuli were displayed against a black background within a white, $14^{\circ} \times 11.3^{\circ}$ frame. As task cues, the label Color was shown in the upper left corner, and the label Shape was shown in the lower right corner. Each word was about $1.1^{\circ}$ wide $\times 0.5^{\circ}$ high. A thin white frame (1 pixel) surrounded each task label; it thickened ( 8 pixels) for the label corresponding to the currently relevant task. The task stimulus was presented centrally, $2.9^{\circ}$ from the bottom of the frame. The direct distance between the task labels and the stimulus was $9^{\circ}$. The stimulus was either a red or green rectangle with a side length of about $1.2^{\circ}$.

Design and Procedure. To keep the experimental session a manageable length, we included only single-task and fade-out blocks. Throughout all of the blocks, task cues were present for the first 40 trials. For two single-task and two fade-out blocks - one block for each task - task cues disappeared from Trial 44 onward. For the other two single-task and fade-out blocks, the task cues remained, as in Experiment 1. On no-cue fade-out blocks, Trials 41 through 43 served as a "transition phase," during which the participants had an opportunity to adapt to the changed task demands signaled by the crossed-out task cue, which was no longer relevant for the remainder of the block. Each individual test block was preceded not only by detailed instructions, but also by a 14-trial practice block that resembled the structure of the following test block in a compressed manner (initial phase, 5 trials; transition phase, 3 trials; fade-out phase, 6 trials). The sequence of the eight test blocks was counterbalanced according to a Latin square.

Each trial began with a 500-msec period during which only the stimulus frame was present. Then the task cue was presented for $100 \mathrm{msec}$, followed by the stimulus. The cue and stimulus remained on the screen until the correct response was entered. The two tasks 
required participants to respond to either the color (red or blue) or the form (vertical or horizontal) of the stimulus, and the participants made their responses using the "/" and " $\mathrm{z}$ " keys of the keyboard.

\section{Results}

RTs were filtered as in Experiment 1. Error rates for the critical fade-out block were again below 5\% for both groups. ${ }^{2}$ We looked at correct responses from Trial 44 onward, breaking down the block into five 10-trial segments. Figure 3 shows the relevant results. Older adults, but not younger adults, again showed large fade-out costs when task cues remained present during the entire fadeout phase. Fade-out costs were substantial but lower than in Experiment 1. We attribute this difference to the fact that, overall, the participants had received considerably more practice in Experiment 2 than in Experiment 1. Consistent with the visual-routine hypothesis, Figure 3 shows that older adults' cost almost disappeared when no cue was present during the fade-out phase. Older adults' performance was clearly more influenced by the presence/ absence of the cue than was the performance of younger adults, age $\times$ task type (fade-out vs. single task) $\times$ cue presence $[F(1,46)=18.02]$. Separate analyses showed that older adults were considerably better without than with task cues $[F(1,46)=15.11]$, whereas younger adults showed, if anything, the opposite trend $[F(1,46)=2.94$, $p=.10]$. The critical fade-out $\times$ cue interaction was reliable for the final segment in older adults $[F(1,23)=6.86$, $p<.05]$, but younger adults showed the opposite trend $[F(1,23)=3.30, p=.08]$.

\section{CONCLUSIONS}

Switching flexibly from a high- to a low-control mode of processing is easy for a younger adult's cognitive system, but poses considerable difficulties as we grow older (Mayr \& Liebscher, 2001). Studies of eye-movement patterns during complex visual-motor tasks have suggested that people will often minimize the use of internal representations to guide action selection (Ballard, Hayhoe, \& Pelz, 1995). Rather, they rely on task-specific visual routines that extract the relevant information from the visual scene as needed, treating the environment as an external memory (e.g., Ballard et al., 1997). These studies also suggest that the relative reliance on internal versus external information is a delicate optimization problem. Here, we show that older adults solve this optimization problem differently from younger adults.

In our paradigm, the critical information was very simple - namely, which of two tasks remained relevant after an initial task-switching phase. As indicated by small cuefixation times and fade-out costs, younger adults seemed to rely almost exclusively on the internal task set representation. In contrast, older adults continued to inspect the cue even though the cue information was completely redundant with a possible internal representation. Cue inspection was almost entirely responsible for the large RT fade-out costs. Of course, external and internal information is redundant for older adults only if they are capable of establishing an adequate internal representation of the current task demands. Thus, it was revealing that older

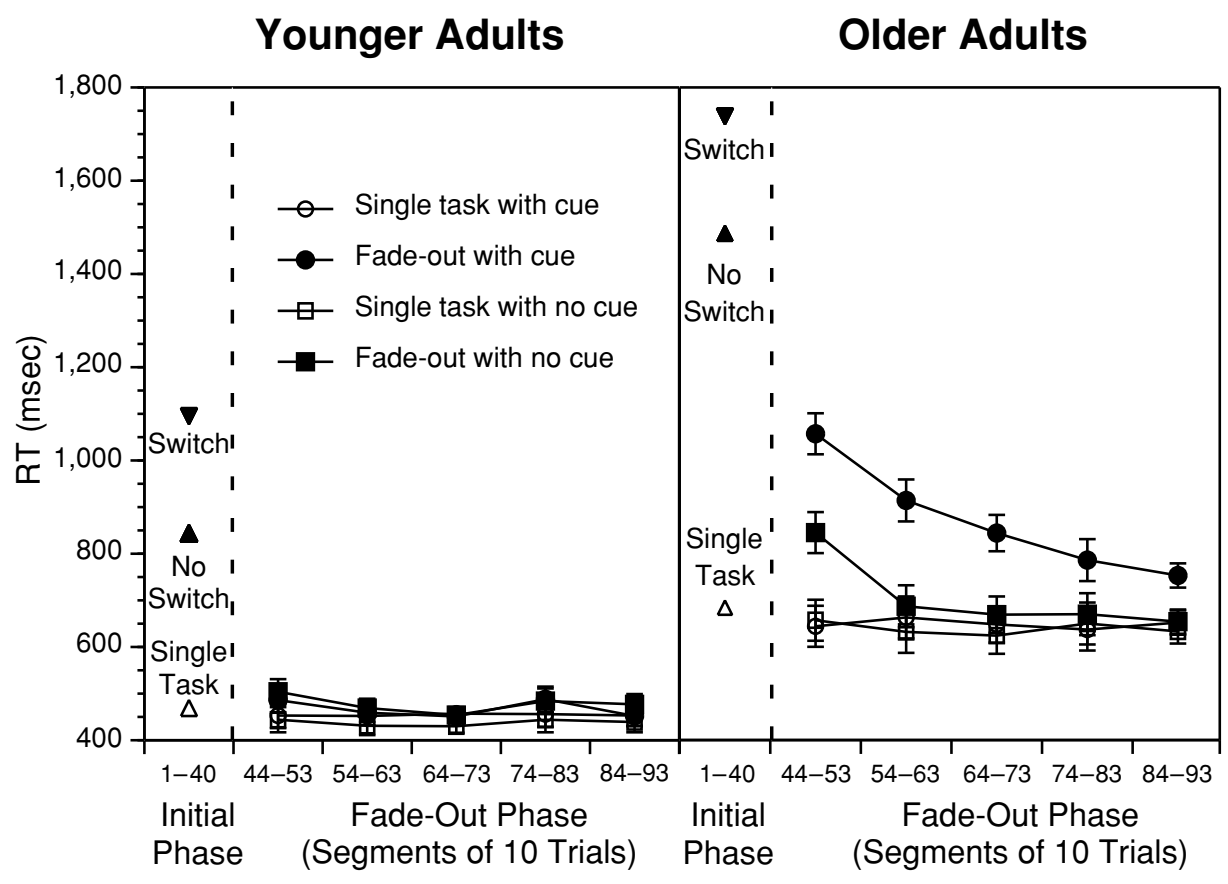

Figure 3. Older and younger adults' average RTs for six 10-trial segments of the fade-out phase, computed separately for the conditions with and without cue and for the corresponding segments of the single-task conditions. Error bars represent the $\mathbf{9 5 \%}$ within-subject confidence interval for the interaction between the fade-out and the cue factor, computed separately for each segment. 
adults' fade-out costs were almost eliminated when individuals were forced to rely on internal representations because task cues were removed in the fade-out phase. It is not the faulty internal representation that causes persistent use of the task cues; rather, the presence of task cues triggers cue inspection, and in turn, is responsible for the behavioral costs during the fade-out phase. Similar tendencies of older adults to rely on external versus internal information have also been reported from studies using a more complex paired-associate learning task (Rogers, Hertzog, \& Fisk, 2000). The critical difference here is that older adults' specific information-use strategy has profound effects in a simple executive-control situation in which the amount of information to be represented internally is extremely limited (essentially 1 bit).

The fact that both younger and older adults can perform the task in the absence of external cues suggests that less reliable internal representations are not the immediate cause of older adults' persistent use of these cues. However, generally noisier internal context representations (see, e.g., Braver et al., 2001) could be an indirect factor behind older adults' increased tendency to use external information. One way of adapting to noisier internal representations is to buttress these representations via external information sources. Indeed, there may even be a nonspecific increased tendency to consult the environment in older adults: In the single-task control blocks, there was also a higher rate of inspecting the cue $(24 \%$ of trials, in comparison with $6 \%$ for younger adults; see Figure $2 \mathrm{~B}$ ). ${ }^{3}$ However, the more striking result was that older adults' tendency to rely on the task cues was dramatically increased in the immediate aftermath of a phase in which task cues were critical for performance.

There are two ways in which a bias toward external information sources may have produced such a pattern of results. Older adults may have a generally reduced threshold for using external sources of information. An external signal that, in the immediate past, was critical for performance (i.e., the task cues during the task-switching phase) may possess greater saliency and thus, with greater probability, surpass older adults' lower threshold needed to draw attention in comparison with a signal that was less recently critical for performance (e.g., the cues in the single-task blocks). Alternatively, older adults' cue-inspection behavior during the fade-out phase may be an aftereffect of their greater reliance on cue processing during the initial taskswitching phase. For example, Logan's instance theory (1988; see also Mayr \& Bryck, 2005) suggests that the constellation of processes applied in a particular stimulus situation are encoded as memory instances and that the quality of these instances is dependent on how much attention was applied during the selection situation. If older adults allocate more attentional resources to the initial task-switching phase than do younger adults, they may sample more persistent memory records that then continue to be retrieved automatically when similar stimulus situations recur within that fade-out phase.

Whatever may turn out to be the precise mechanism underlying older adults' increased cue utilization, the present results are important because they establish a significant shift of information processing in demanding situations across the life span. When external sources of information are present, older adults are much more likely than younger adults to use them, even if the information demands are very limited and can be represented reliably, and even if the use of the external sources incurs a behavioral cost. Note that we have shown that these age differences are directly associated with age differences in the use of environmental information. Often, general theories of cognitive aging attempt to account for age differences in performance across a wide range of tasks by positing a fundamental change such as a general slowing of processing (see, e.g., Cerella, 1990). Such theories typically predict increased effects of experimental manipulations in older adults than in younger adults. The present results are inconsistent with such generalized slowing accounts precisely because we have shown that the presence of age effects (e.g., fade-out costs) are controlled by the availability of environmental cues. Thus, the causal factor that determines the presence of an age difference is the availability of environmental information. Because general slowing accounts eschew specific process accounts for age differences, these theories are ill-suited to explaining such context-specific age effects.

At this point, we withhold judgment about the degree to which older adults' reliance on environmental information should be interpreted as a deviation from optimal performance in comparison with a younger adult's performance. What presents itself as a cost under some circumstances may actually indicate a beneficial adaptation that compensates for the pitfalls of an overall less reliable system (see, e.g., Baltes, 1997).

\section{REFERENCES}

Ballard, D. H., Hayhoe, M. M., \& Pelz, J. B. (1995). Memory representations in natural tasks. Journal of Cognitive Neuroscience, 7, 66-80.

Ballard, D. H., Hayhoe, M. M., Pook, P. K., \& Rao, R. P. N. (1997). Deictic codes for the embodiment of cognition. Behavioral \& Brain Sciences, 20, 723-767.

BALTES, P. B. (1997). On the incomplete architecture of human ontogeny: Selection, optimization, and compensation as foundation of developmental theory. American Psychologist, 52, 366-380.

Braver, T. S., Barch, D. M., Keys, B. A., Carter, C. S., Cohen, J. D., KAYE, J. A., ET AL. (2001). Context processing in older adults: Evidence for a theory relating cognitive control to neurobiology in healthy aging. Journal of Experimental Psychology: General, 130, 746-763.

Cerella, J. (1990). Aging and information-processing rate. In J. E. Birren \& K. W. Schaie (Eds.), Handbook of the psychology of aging (3rd ed., pp. 201-221). San Diego: Academic Press.

Kray, J., \& Lindenberger, U. (2000). Adult age differences in task switching. Psychology \& Aging, 15, 126-147.

Logan, G. D. (1988). Toward an instance theory of automatization. Psychological Review, 95, 492-527.

MAYR, U., \& BRYCK, R. L. (2005). Sticky rules: Integration between abstract rules and specific actions. Journal of Experimental Psychology: Learning, Memory, \& Cognition, 31, 337-350.

Mayr, U., \& Liebscher, T. (2001). Is there an age deficit in the selection of mental sets? European Journal of Cognitive Psychology, 13, 47-69.

Rogers, W. A., Hertzog, C., \& Fisk, A. D. (2000). An individual differences analysis of ability and strategy influences: Age-related dif- 
ferences in associative learning. Journal of Experimental Psychology: Learning, Memory, \& Cognition, 26, 359-394.

WeCHSLER, D. (1981). Wechsler Adult Intelligence Scale-Revised. San Antonio, TX: Psychological Corp.

Winer, B. J., Brown, D. R., \& Michels, K. M. (1991). Statistical principles in experimental design (3rd ed.). New York: McGraw-Hill.

\section{NOTES}

1. Error rates across the switch, no-switch, and single-task conditions were $4.0 \%, 3.2 \%$, and $1.6 \%$, respectively, for younger adults and $3.8 \%$, $2.5 \%$, and $2.8 \%$, respectively, for older adults. For the single-task phase of the fade-out block, error rates were $1.1 \%$ and $1.9 \%$ for younger and older adults, respectively.
2. Error rates across the switch, no-switch, and single-task conditions were $8.4 \%, 5.7 \%$, and $3.8 \%$ for younger adults and $19.6 \%, 5.4 \%$, and $2.1 \%$ for older adults. For the single-task phase of the fade-out block, younger adults had error rates of $4.4 \%$ and $4.8 \%$ for cue and no-cue conditions, respectively, in comparison with older adults' $2.4 \%$ and $2.2 \%$, respectively.

3. This result is somewhat ambiguous, because single-task blocks occurred in the larger context of blocks that did involve task switching. Thus, older adults' increased cue-inspection tendency in single-task blocks may either constitute an unconditional overreliance on external information or a manifestation of the same, albeit reduced, cost observed in fade-out blocks.

(Manuscript received November 16, 2005; revision accepted for publication March 17, 2006.) 